bad symptoms; the wound looked healthy, and healed gradually.

In a few weeks, he was allowed to get up; and on May 18th, was discharged cured. He could walk perfectly well; and there was no tendency to hernial protrusion.

\section{Giginal Commaniations.}

\author{
CASES OF STRANGULATED HERNIA,
} TREATED BY INFLATION OF TIIE BOWELS, AND BY SHAKING TIE PATIENT WHILS' IN

AN INVERTED POSITION.

By Richard Griffin, Esq., Weymouth.

CASE I. A short time since, I visited, in consultation, a female, with symptoms of strangulated femoral hernia, which had existed for a couple of days. In consequence of several attempts at reduction, the hernia, about the size of a walnut, had become very painful, and there was some tenderness of the abdomen; romiting was frequent, and there was no action of the bowels, although several large aperient doses and enemata had been administered. I procured a pair of bellows and passed the nozzle into the anus, an assistant holding the nates well together, so as to prevent, as much as possible, the escape of air from the bowels. The patient complained of the distension giving her pain; but the inflation was continued for about a cuarter of an hour, much of the air es. caping eitler through the bellows, or by the side of the nozzle; but it was, apparently, of no benefit.

A fresh consultation was then held; when it was deemed advisable to recommend an operation. This, however, was postponed for a short time, but fortunately was not needed. In about an hour after inflation, the bowels acted freely, and all symptoms of strangulation ceased; the distension having caused the forcible withdrawal of the strangulated knuckle of intestine which had prevented the peristaltic action of the bowels. 'The patient recovered.

CASE II. In another case, to which I was called in consultation, the old woman had stercoraceous vomiting for three or four days, with constipation. The hernia was femoral and irreducible. The bellows were used in this case, as in the last; and, although they appeared at the time to have been of no avail, yet, in about an hour, a free action of the bowels took place. Unfortunately, however, there was no stopping the diarrhœa which ensued, arising from the drastic purgatives which had been administered; and the old lady died in forty-eight hours. The inflation, however, reduced the hernia; and so far was a success.

CASE III. I was called in consultation to a man who had a strangulated insuinal hernia, which could not be reduced by the taxis. Purgatives and enemata had been freely administered during the preceding three days. I at once tried the bellows; which had not been used many minutes, before the man called out, "You are blowing up my purse!" Such was, indeed, the fact; the scrotum having become largely distended with air, owing to a laceration in the rectum having been made with the pipe of the enema-syringe, which, I subsequently ascertained, had been so roughly used, that the man called out when the enema was being administered, and blood followed the withdrawal of the pipe. The bellows were removed, and the man immediately went to the night-stool ; and a copious evacuation followed. I did not see the patient again; but I subsequently heard that he got well, and the air in the scrotum was soon absorbed.

CASE IV. A fourth case has been related in one of my Poor-law pamphlets, in which a stranorulated hernia was reduced by the bellows; the taxis, enemas, and purgatives, having previously failed.

These cases prove that, before the operation for strangulated hernia is performed, it would be well to try the effects of inflation; although this, like everything else, is sometimes liable to fail, as the following cases will prove; superadded to which, shaking the patient whilst in the inverted position, also failed.

CASE v. A man with scrotal hernia, to whom I was called in consultation, had all the usual remedies tried, including inflation of the bowels, but in vain. He was then hoisted with his knees over my medical friend's shoulders, and lifted in that position until only his head touched the bed, and had a thorough good shaking. The intestine, however, was too tightly held to be dragged from its position by this procedure; he was, therefore, obliged to submit to an operation, by which he was cured.

CASE VI. A lady, very stout, about 50 years of age, who had suffered for years with an irreducible umbilical hernia, for which she wore a supporting abdominal-belt, having one day used a little extra exertion, came home fatigued, and complained of pain in the bowels, followed by sickness and constipation. Enemata, salines in a state of effervescence, then opium, and finally purgatives, with inflation of the bowels, were tried, together with the taxis; but the hernia could not be reduced. A former medical at. tenclant of the lady was then summoned from a distance; and, on his arrival, he suggested that shaking in the inverted position should be tried. Accordingly, the patient's knees were placed over his shoulders, with her legs hanging down his back, and, with the assistance of several persons present, he gradually raised himself into an upright position, the patient's head only touching the bed. He then gave her two or three good shakings, which did not occupy more than a minute or two; but, on looking at the patient's face, I discovered that breathing had ceased, and she was pulseless. We instantly placed her in the recumbent position; the window was thrown open; and a napkin, with the end dipped in water, was very freely applied to the face and chest, the slaps from it being anything but gentle. In a few minutes, there was a slight sigh; and, after a short time, we had the pleasure of having our patient in no worse a state than before the attempt at reduction by shaking; but it was felt to be a painfully narrow escape by all present, and may serve as a warning, not to be disregarded by medical men, that they ought to be careful how they turn stout people topsyturvy. A medical gentleman from Bath was telegraphed for ; but, in spite of the efforts of four surgeons, our patient gradually became worse, and died. As we were not permitted to have a post mortem examination, the precise cause of death was not ascertained.

\section{HYDROCELE.}

By John Thompson, M.D., F.R.C.S., Bideford.

HYDROCELE is of such common occurrence, that it must be a subject of interest to every practitioner. It affects persons of all ages, but is most frequent in the later periods of life. I know that this will be disputed; but I am nevertheless persuaded that, if it be taken into consideration how small is the number of persons living between the ages of fifty and seventy, compared with those between the ages of thirty and fifty, experience will justify my opinion. 
Little need be said of the symptoms which almost invariably distinguish the disease. The pyriform shape, semi-elastic fluctuating feel, translucency, and increase of the tumour from the bottom upwards, are familiar indications. Other attendant marks, such as a feeling of weight in the scrotum, with dragging pains along the loins, are commonly present. It is curious to notice how every symptom may vary-the shape may be round, oroil, or very much like the outline of the ram's testicle; the tumour may be almost as hard as a cricket-ball, and pain in the back be also present, equal to that felt where organic disease of the testicle exists. I mention the two last points in connexion, because one might hastily infer from them, that diseased testicle and not hydrocele was present, an error which $I$ have known to occur. The degree of the translucency is said to vary much; but perhaps this assertion may rest somewhat on the care or otherwise with which the examination is made. Very little practical direction is usually given in books on this point; but Mr. Erichsen very properly recommends the surgeon to grasp the back of the tumour, so as to tighten the integuments, before passing the light through them.

I am satisfied that, if this plan be constantly adopted, but little difficulty will be experienced in applying this most diagnostic test, and obtaining a satisfactory result. The rays of light from a window oir candle should pass laterally through the swelling, the latter being held off so as to insure this; thus the testicle and appendages which lie behind may be avoided.

Where the patient consents, which he generslly will, and nothing forbids the operation for the radical cure, I presume all are agreed that this should be effected; but in a great number of persons afflicted, age, or the existence of organic disease, render it inapplicable. Under these circumstances, it is usual to adopt what is termed the palliative treatment, and to tap the swelling three or four times a year with a trocar and cannula, on which I wish to offer some comment.

It is admitted that this treatment offers but little hope of cure, the instances where it has occurred being very rare; but there is another method of dissipating the tumour that is not unfrequently attended with a radical cure of the disease; I mean the method by acupuncture.

In the congenital hydrocele of children, it will very generally succeed, and not unfrequently in that of adults. I admit its great inferiority to the practice by injection; but where this cannot be adopted, as in children and old persons, it seems to me but a common-sense thing to use the method next in order of efficacy.

For many years, I have not tapped a hydrocele with a trocar, unless as a prelude to injection; but have, in all cases, used acupuncture, and, to mv mreat gratification, every now and then a cure is effected, with less inconvenience to the patient than had he been trpped as many times in the ordinary way.

It is no new surgestion to treat hydrocele thus, as it was long ago tried in Germany, and since in this country by Mr. R. Keates and Mr. Travers, who ieported that "it acted favourably in a number of cases, but was not generally to be relied on."

I beliere this is quite as much as can be said foi it. My position is simply, that, as acupuncture properiy performed gives the patient even less inconvenience than tapping with the trocar, and is frequently followed by a radical cure, which the operation by tapping is not, therefore it is advisable to use acupuncture in all those cases that are unsuitable for the treatment by injection, seton, etc.

There is something to be said about the manner in which acupuncture is to be performed. It is advised by a recent writer to use a grooved needle, but for what reason, I cannot understand. If the needle is cylindrical in the shaft, with a conical point, as is usually the case, it is a very unscientific shape for puncturing tight structures like the skin and sac. All needles intended to penetrate the human structures reacily, should be either triangular or spearshaped at the puncturing extremity; and the shaft should not be large. I always use a needle that Mr. Listen employed in the operation for hare-lip; it has an expanded spear-shaped extremity for piercing, and a small cylindrical shaft. From three to six punctures may be made with this, well through the skin and sac, the scrotum being grasped firmly on its hinder part in the left hand at the same time, so as to tighten the integuments. No marked pain is felt; a little fluid oozes from the punctures while the hand is applied, but ceases on its removal; in a day or two the tumour is gone.

The needle used by the sewers of kid gloves will answer equally well with that I have described, it has a sharp triangular extremity for piercing; our needle-makers knowing that this is the form necessary for entering tough animal structures, the round conically pointed needle is only applicable for loose textures.

\section{茎e}

Lectures on the Diseases of Womme. By Challes West, M.I)., Fellow of the Royal College of Physicians; Examiner in Midwifory at the University of London, etc. Third edition. Pp. 687. London: 1864.

$\Lambda$ Pontrox of the first edition of this work was issued by Dr. WEsT more than eight years ago; and it was then described by him as "embodying the results of ten years of observation in the wards of a hospital." Since that time, two editions have appcared; one in 1858 , and the other in the present year-being the work now before us. 'The author has, he says, "carefully revised the whole work, and arlderl to it whatever larcer experience has increased his knowledge."

The thirty-three lectures of which the book consists are distributed as follows. Lectures 1 and $\psi$ are on the Symptoms of 1)iseases of Women; 3,4 , and 5 , on Menstruation and its Disorders ; 6,7 , and 8 , on Inflammations of the Uterus; $9-13$, on Misplacements of the Cterus; $14-18$, on Cterine 'Iumours and Outgrowths; 19,20 , and 21 , on Malignant or Cancerous Diseases of the Uterus; 22 and 23 , on Inflammation of the Uterine Appendages, and Uterine Hæmatocele; 24, on Inflammation of the Ovaries; $25-30$, on Ovarian 1)ropsy; 31, on 1)iscases of the Female Bladder; 32 , on Diseases of the Urethra and Vagina; and 33, on Diseases of the Exterual Organs of Generation.

This book has been so long before the profession, and must be so well known, that to subject it to the oriinary process of reviewing seems almost umnecessary. Yet, believing that there are some anong our readers who would desire to know the opinions of so eminent an authority as Dr. West, we will endeavour to gratify them by giving an outline of his doctrines on various more or less important topics.

The Speculum. IDr. West describes, in the second chapter, the uterine speculum, and gives rules for its 